\title{
ASPECTOS ESTRUCTURALES SOBRE EL COMPORTAMIENTO SISMICO DE TORRES, TANQUES ESBELTOS, CHIMENEAS Y TANQUES ESFERICOS
}

\author{
Neftalí Rođríguez Cuevas (I) \\ 1. INTRODUCCION
}

Para el diseño estructural de las instalaciones industriales, bajo la acción de sismos intensos, es necesario evaluar los daños que se presentan en ellas, a fin de establecer los criterios que permitan su diseño bajo esas solicitaciones. En este trabajo se resume la revisión de daños en zo nas sísmicas publicados en la literatura y se analizan las causas que ori ginaron daños en tanques esbeltos, torres de diversos tipos, chimeneas y tanques esféricos.

Se establecen los criterios seguidos en diversos países para revisar los efectos de movimientos del terreno, poniendo especial énfasis en el re sultado de planteamientos comunes para el análisis sísmico y los resulta dos de pruebas en mesas vibradoras, a fin de establecer las principales di rectrices del diseño de estructuras, de los tipos comúnmente usados en ins talaciones industriales.

Se presenta la bibliografía revisada a fin de establecer los aspectos de diseño sísmico que se condensan en el escrito.

\section{DANOS DOCUMENTADOS EN LA LITERATURA}

Se describen las experiencias obtenidas durante inspecciones en zonas sísmicas, en las cuales se presentaron daños importantes, poniendo especial énfasis en detectar las características de los daños.

En la ref 1, se establecen con claridad los efectos de dos sismos in tensos en China: uno con magnitud 7.3 en la escala de Richter en el áreá de Haichén, que ocurrió el 4 de febrero de 1975 y otro de magnitud 7.7 en la escala de Richter, en la región de Tangshan que ocurrió el 27 de julio de 1976.

El sismo de Tangshan, que ocurrió en el Noreste de China el 27 de julio de 1976, resultó ser uno de los sismos recientes que mayores pérdidas de vidas y propiedades ha provocado, estimándose que 650000 personas perdie ron la vida por su causa. Resultó de un sistema complejo de eventos entre placas, ref 2, que generó un movimiento de magnitud 7.7 en la escala de Richter, con un sismo posterior, de magnitud 7.2 en la misma escala, que se presentó $15 \mathrm{hs}$. después del evento principal, cuyo epicentro se locali zó a menos de $10 \mathrm{~km}$ de Tangshan, mientras que el sismo principal se origí nó a una distancia de $20 \mathrm{~km}$ (USGS).

(I) Ingeniero Consultor, Miembro Sociedad Mexicana de Ingeniería Sísmica 
En estos dos sismos, los daños en las instalaciones industriales varia ron entre diferentes grados de intensidad, pero las estimaciones estadisti cas en las zonas de alta intensidad, mostraron que los daños afectaron más del cincuenta por ciento del equipo industrial.

Entre las estructuras dañadas se encontraron torres, tanques y hornos usados para tratar o almacenar combustibles, líquidos venenosos o explosi vos y gas.

Durante la revisión de los daños ocasionados en ambos sismos, se obser vó que los dañols se debían a dos causas principales: el colapso de los ed ficios que albergaban al equipo, o la destrucción del apoyo en la cimenta ción de los equipos. Estas dos causas provocaron el aplastamiento de las instalaciones industriales al caer las partes derribadas del edificio, la inclinación o colapso del equipo; la rotura de tubos de conexión, cuando se presentó la licuación del suelo, o se generaron hundimientos diferencią les importantes durante los sismos. Estas últimas causas provocaron que las anclas se alargaran excesivamente, o se presente su falla por la acción del cortante sísmico. La falla de los edificios no se trata en este traba jo, sino que la atención se concentra en la falla de la base de los equipos industriales.

\section{Daños en torres}

Existen ejemplos específicos como el de una torre de enfriamiento de $10 \mathrm{~m}$ de altura y $2 \mathrm{~m}$ de diámetro, soportada por 12 anclas en su base, de las cuales nueve fueron arrancadas durante el sismo. No se encontró daño en la torre misma por efecto del sismo.

La repetición de este problema en varias torres conduce a pensar que el efecto más importante de la acción del sismo en las torres, se refleja en el cuidado tomado en el diseño de la cimentación y de las anclas de so porte, por lo que resulta indispensable conocer el momento de volteo en Ia base y el cortante basal, como un criterio adecuado para la revisión de los efectos sísmicos en este tipo de torres, más que efectuar análisis muy elaborados de la torre misma.

Esta afirmación se sustenta en el resultado de anâlisis sísmico, basa dos en el diseño espectral, que valuaron los esfuerzos en las paredes de las torres; se encontró que ellos resultaron ser de pequeña magnitud, dado que el diseño sigue recomendaciones mecánicas que limitan los espesores, los cuales resultaron adecuados para soportar la acción sísmica. Este re sultado se corroboró al revisar los daños en las torres dañadas en las dós regiones chinas arriba mencionadas, observándose que ellos eran atribuibles a un mal diseño de las anclas. Como resultado de esta revisión en diez torres, el análisis dinámico mostró que el periodo del primer modo, se en contraba comprendido entre $0.2 \mathrm{seg}$ y $0.7 \mathrm{seg}$, cuando la relación $H^{2} / D$ osci laba entre 40 y 400 , siendo $H$ la altura de la torre y $D$ el diánetro exte rior, con relaciones $H / D$ comprendidas entre $3 y 5$, lo cual indicó que se trata de estructuras relativamente rígidas. 
Estudios con el método de elementos finitos de ellas, y pruebas en me sa vibradora realizados en China, mostraron que la aparición de un sismo de magnitud 7, provocaba esfuerzos en las paredes, inferiores a los origi nados en los mismos puntos por la acción de un viento de $90 \mathrm{~km}$ por hora en zonas costeras, por lo que su diseño queda controlado por la acción del viento, más que por la acción sísmica, a la cual resultan ser suficientemen te tenaces para soportarla.

En algunas torres que contenían líquidos, la presión del líquido contra las paredes resultó ser mucho mayor que la fuerza de inercia de los tanques. Housner (ref 3) ha dividido la presión dinámica del fluido en presión con vectiva y presión de impulso. Cuando el periodo predominante del movimien to del suelo es cercano al periodo del líquido, la presión convectiva jueğa un papel importante, pero esta condición no se presenta con frecuencia, si no que resulta ser el caso excepcional; en la mayoría de los casos, la pré sión del impulso es predominante (ref 4) por lo que la acción de ella en la revisión de las paredes, siempre debe ser considerada, tal como se estable ce en la ref 5 .

Bajo la presión dinámica del fluido, originada por sismos, los esfuer zos en la pared de tanques bien diseñados son pequeños, pero la aparición de esfuerzos cortantes importantes, en combinación con los esfuerzos de mem brana, puede inducir la aparición de pandeo y abolsamiento de las paredes, como se detectó en algunos tanques de Haichen y Tangshan, así como daños en las anclas de soporte perimetral.

Daños en altos hornos

Los tipos de altos hornos usados en instalaciones industriales pueden ser muy diferentes, por lo que sólo se comentan en este escrito los altos hornos de acero, comúnmente usados en planta petroquímicas y los altos hor nos metálicos, que se encuentran en planta siderúrgicas.

Los altos hornos de plantas de acero y fundición son altos y pesados, e investigadores en varios países ( $r e f 6$ y 7 ) se han interesado en mejorar su capacidad para resistir la acción de sismos.

La estructura de estos hornos se puede dividir en dos partes: el cuer po y la parte superior. La parte superior incluye los tubos de salida y las instalaciones auxiliares en la parte superior. Los altos hornos gran des tienen un marco superior para soportar el peso de la parte alta, que permite sostener a los recuperadores de polvo.

En Japón, diseños resistentes a la acción de sismos han conducido a la construcción de marcos externos al horno para soportar las solicitaciones sísmicas, seleccionándose los altos hornos autoportantes en áreas de baja intensidad y el de anillos y largueros soportados, en áreas de sismicidad

intensa. Estos tipos de hornos ya han soportado un sismo en Chile, en ma yo de 1960 y en Tokochichu, en 1968. Los altos hornos chinos no han sido diseñados contra la acción sísmica, y durante los sismos de Haicheng y 
Tangshan se presentaron daños que consistieron en:

a) El acero fundido se solidificó en las cámaras interiores en varios hor nos de pequeña capacidad $\left(100 \mathrm{~m}^{3}\right)$ debido al corte de electricidad y agua.

b) En zonas de mayor intensidad, cuatro pequeños hornos resultaron dañados. En el primero se presentó pandeo de las paredes. En otro, la soldadu ra entre un tubo de salida y el cascarón del horno se agrietó y se ge neró pandeo por exceso de esfuerzos. El desplazamiento de la parte su perior fue, tan amplio que dañó las instalaciones auxiliares ahí coloca das.

c) En áreas dé menor intensidad, 11 hornos con capacidad superior a mil metros cúbicos resultaron dañados en la parte superior, pero no se en contró daño en el cuerpo del horno.

A fin de entender los daños, se realizaron pruebas en un modelo de hor no con $1513 \mathrm{~m}^{3}$ de capacidad, del tipo autoportante (ref 8) encontrandose que los esfuerzos en las paredes verticales fueron pequeños, mientras que el factor de seguridad contra volteo resultó ligeramente superior a 1. Los desplazamientos en la parte superior del modelo fueron cinco veces mayores que los del cuerpo del horno y se observó que cuando se coloca una gasa en la tubería de salida y se trasmite la carga total de las instalaciones en la parte superior, a un marco de soporte, se tiene un buen diseño antisismi co.

Daños en chimeneas

Este tipo de estructuras ha resultado particularmente sensible a la acción de sismos, y presentan daños con frecuencia durante sísmos intensos. Durante el sismo de San Fernando, Cal. de magnitud 6.6 que ocurrió el 9 de febrero de 1971, los daños fueron cuidadosamente clasificados (ref 9) en dos áreas de Glendale, seleccionandose dos áreas principales de observación. En el área 1, se contaron 494 edificios en total y 325 tenían chimenea. En el área 2 se contaron 549 edificios y 285 tenían chimenea. Después del sis mo, en el área 1 se contaron 135 chimeneas dañadas, mientras que en el área 2,77 de ellas, resultaron dañadas.

Esta investigación mostró que el daño se concentró principalmente en chimeneas, debido a los efectos combinados de degradación del material con el tiempo y a un mal diseño inicial.

En otros sismos, como por ejemplo en Chile, Estados unidos o Perú, se han observado daños en estructuras de chimeneas industriales de acero, con creto $y$ de tabique, consistentes en pandeo de lás paredes, daños en las anclas, volteo y rotura del fuste. 
Dada la alta incidencia de daños en las estructuras descritas, se pre senta más adelante un breve resumen de los esquemas de cálculo usados para el diseño de este tipo de estructuras.

\section{CRITERIOS PARA DISENO DE TORRES Y EQUIPOS}

Desde 1957 existen recomendaciones publicadas para diseño en regiones sísmicas, de edificios industriales y sus instalaciones. En la ref 10, se muestran los criterios soviéticos para el análisis, proponiendo la expre sión siguiente para valuar las fuerzas en las masas de una estructura

$$
s_{k}=Q_{k} k_{c}^{\alpha}
$$

donde $S_{k}$ la fuerza de diseño en cualquier punto $k$, donde se presenta la ma
sa $l_{k}$

$Q_{k}$ la fuerza generada por las fuerzas de inercia

k el coeficiente sísmico

$\alpha \quad$ coeficiente dependiente de las características dinámicas

Los valores de $\alpha$ para torres lo definen igual a 2 en la parte superior de la estructura, e igual a 1 en la base, con interpolación lineal a lo lar go del fuste. Para las conexiones rígidas entre los elementos de la es tructura, del tipo de anclas, las recomendaciones establecen un valor de $\alpha=5$, lo cual muestra un excesivo cuidado en sobrediseñar las anclas, a fin de evitar daños en la estructura que soportan.

Las recomendaciones SEAOC (ref 11) definen el cortante basal, su dis tribución a lo alto de la estructura y los momentos que se generan para es timar el volteo. En la fig 1 se condensan las recomendaciones a este res pecto, notándose que la estimación del cortante basal involucra un coefi ciente de la estructura $K=2$ para torres y chimeneas, $y$ considera las carac terísticas dinámicas de la estructura y la intensidad de la acción sísmica, a través del coeficiente $Z$, que aparece en la expresión:

$$
\mathrm{V}=\mathrm{ZKCW}
$$

donde

V cortante en la base

Z coeficiente de sismicidad

K coeficiente de estructura 


$$
C=0.05(T)^{-1 / 3}
$$

$T$ periodo fundamental de la estructura

w peso de la estructura

En los procedimientos de SEAOC no se hace notar la influencia del tipo de cimentación, pero Rinne (ref 12) propone un coeficiente S que considere las condiciones del suelo, variando entre 1 para suelo rocoso y valores ma yores a 1, para suelos suaves, dependiendo del tipo de estructura.

La selección del coeficiente $K=2$ para torres $y$ chimeneas, difiere de los valores $2 / 3$ y $4 / 3$ propuestos como límites para edificios comunes, lo cual se atribuye al hecho de ser "estructuras simples".

En los criterios chinos se recurre a planteamientos similares a lo es tablecido por las recomendaciones soviéticas, proponiendo valuar el cortan te en la base $Q_{0}$, mediante:

$$
Q_{0}=C_{2} \alpha_{1} W
$$

donde

$C_{2}$ es el coeficiente de efectos combinados, afectado por el amortigua miento, la ductilidad y el daño experimentado durante temblores

$\alpha_{1}$ coeficiente de efecto sísmico

$w$ peso total del equipo

Al coeficiente $C_{2}$ lo hacen variar entre 0.3 y $0.5, y$ al coeficiente $\alpha_{1}$ lo hacen depender de un espectro de diseño, cuyo valor máximo para intensi dad 8 (similar a MM) lo hacen igual a 0.45 . Recomiendan el método de aná lisis modal para obtener la solicitación sísmica.

En Estados Unidos, han aparecido recientemente recomendaciones para de finir la acción sísmica en equipos industriales (ref 13), en las cuales es tima la acción sísmica mediante:

$$
F_{p}=A_{v} C_{c} P a_{c} a_{x} W_{c}
$$

donde

$F_{p}$ fuerza sísmica aplicada en el centroide del equipo

$A_{v}$ parámetro relacionado con la aceleración máxima correspondiente a la velocidad máxima efectiva durante un sismo

c coeficiente sísmico 
P factor dependiente del funcionamiento del equipo

$a_{c}$ factor de amplificación dependiente del tipo de apoyo

a factor de amplificación relacionado con la variación con la altura de la respuesta estructural $=1.0+h_{x} / h_{n}$

$h_{x}$ altura del nivel $x$ sobre la base

$h_{n}$ altura máxima del equipo sobre su base

En este enfoque ya se hace aparecer a las variables principales obser vadas durante sismos intensos, pero es necesario efectuar una investigación más amplia a fin de definir los parámetros con mayor confiabilidad.

Se puede mencionar en este aspecto, a los estudios de estructuras de torres y tanques esbeltos mediante el método de elemento finito, que han proporcionado información sobre el comportamiento de ellos bajo la acción de sismos registrados, y ya existen algunas soluciones (ref 14) que mues tran resultados coincidentes con los obtenidos de pruebas en modelos cons truídos en mesas vibradoras.

Esta información indica que la idealización mediante el método de ele mento finito de los cascarones que forman la estructura resistente de torres, permite estimar las fuerzas unitarias de membrana y los momentos flexionantes en ellos, con suficiente claridad, pero esos resultados han mostrado también que la acción de los siśnos no induce solicitaciones de muy alta magnitud en las paredes de los recipientes.

En las figs 2 y 3 se muestran los elementos mecánicos obtenidos del análisis de un tanque cilíndrico cubierto con un domo que contiene un líqui do, sometido a la acción de un sismo (PSEQGN), con aceleración máxima nor malizada igual a 0.5 . Se observa que se presentan concentraciones impor tantes de momentos flexionantes en los bordes de la pared vertical, pero en el resto de ella, los esfuerzos son prácticamente nulos.

La solución también muestra que en la parte superior del domo que cubre al tanque, se presentan momentos del orden del doble de los que se presen tan en la base de la pared del tanque. La distribución de fuerzas de me brana asemeja a aquella correspondiente a la distribución máxima de presío nes, obtenida para el mismo sismo en mesa vibradora, pero su orden de mag nitud induce niveles de esfuerzos en la pared relativamente bajos.

La existencia de fuerzas de compresión importantes, inducidas por el sismo, es indicativa de la posibilidad de pandeo de la pared del tanque, como lo demuestra la repetición de este daño en estructuras cilíndricas. 


\section{TORRES DE ENFRIAMIENTO}

Es en este campo en donde se han hecho avances significativos, ya que la industria eléctrica emplea hiperboloides de revolución apoyados sobre columnas desplomadas que forman una retícula triangular de enfriamiento en sus torres.

Gould publicó en 1967 un primer procedimiento de análisis de este tipo de estructuras ( $r e f$ 15) donde definió la manera de obtener los esfuerzos de membrana en mantos de hiperboloide de revolución, sometidos a aceleracio nes sísmicas; las condiciones de frontera no consideraban a los elementos que normalmente refuerzan a estas estructuras en sus bordes, por lo que ese análisis sólo permite tener una primera idea sobre la manera de analizar a las torres.

Posteriornente completó la solución (ref 16 y 17), en donde ya incorpo ra los efectos de las trabes de borde y de las columnas de soporte, que contribuyen de manera importante en la respuesta.

El modelo de análisis lo reduce a un análisis de elementos finito de alta precisión, de cascarones de revolución. Introduce a las trabes de bordes como elementos cortos de mayor espesor, y al sistema de columnas de apoyo lo representa como un elemento rotacional de cascarón, considerando una distribución uniforme de las columnas alrededor de la circunferencia inferior de la torre; en estos elementos considera los efectos de fuerza axial y flexión.

Efectúa un análisis dinámico (ref 18 y 19) expandiendo en serie de Fourier a las variables dependientes a lo largo de la dirección cincunfe rencial, mientras que en dirección meridional selecciona polinomios de comparación de tercer orden, en unión de un procedimiento de condensación cinemática, a fin de llevar a cabo un análisis de vibraciones libres.

Conocidas las características dinámicas de la torre, encuentra las ace leraciones nodales máximas mediante el método espectral de respuesta y cal cula los tres desplazamientos ortogonales de cada elemento del cascarón. Para ello, acepta el concepto de ductilidad comprendido en el espectro, pe ro debido a que los elementos de soporte se encuentran sometidos a flexión y fuerza axial, es necesario llevar a cabo una cuidadosa investigación de los detalles de refuerzo, a fin de que la estructura desarrolle las con diciones de ductilidad supuestas.

El análisis permite obtener las tres componentes ortogonales del des plazamiento de cada elemento, y mediante relaciones constitutivas represen tativas, logra definir las fuerzas de membrana, los momentos flexionantes y torsionantes en todos los elementos del cascarón que constituyen el man to de la torre de enfriamiento. Asímismo obtiene los elementos mecánicos en las columnas de soporte, con lo cual resuelve el problema de análisis de este tipo de torres de enfriamiento. 
Los resultados de este tipo de análisis, en un cascarón con altura cer cana a $120 \mathrm{~m}$ y $100 \mathrm{~m}$ de diámetro en la base, muestran que se trata de una estructura muy flexible, ya que la frecuencia del primer modo es próxima a $2.3 \mathrm{seg}$, mientras que el segundo modo vibra con una frecuencia de 3.8 seg, cuando se consideran las condiciones de frontera reales del cascarón.

La aplicación del análisis espectral muestra que el sistema de columnas funciona como un piso deformable que trasmite la perturbación originada por el movimiento del terreno, y los elementos mecánicos máximos se locali zan en la vecindad de la conexión de las columnas a la trabe de borde infe rior.

Los esfuerzos cincunferenciales resultan ser importantes en la vecindad del borde y tienden a disiparse a medida que se aumenta la altura sobre el borde el cascarón. Los momentos flexionantes cerca del borde presentan va lores muy altos, pero rápidamente disminuyen al alejarse de la trabe de bōr de.

Los desplazamientos horizontales son altamente dependientes de la rigi dez de las columnas inferiores y los desplazamientos máximos en la parte sú perior del cascarón resultan ser del orden de $10^{-4}$ veces la altura del cas carón; la configuración deformada presenta dos cambios de curvatura en la parte cercana a las trabes de borde.

Solamente existe este análisis de las torres, idealizados como mantos de cascarón, sin que haya sido establecido algún procedimiento simplifica do de análisis, para calcular la respuesta de estas torres, bajo la acción de un sismo de diseño.

\section{CHIMENEAS}

El estudio del comportamiento sísmico de chimeneas ha sido ampliamente desarrollado en diversas partes del mundo, (ref 20, 21 y 22), 10 que permi te diseñar este tipo de estructuras.

La revisión de los criterios que se han propuesto, permitió establecer tres procedimientos de análisis de ellas, que dependen de las característi cas de la estructura y del terreno de cimentación. Para discriminar entre ellos, es necesario definir los parámetros i y j; i, representa la rela ción entre la rigidez a desplazamjento horizontal de lá base de la chimenea, a la rigidez lineal de la estructura; j es la relación entre la rigidez an gular de la cimentación, a la rigidez angular de la chimenea.

Cuando los parámetros $\mathbf{i}$ y $\mathbf{j}$ son mayores a 10 , es posible realizar un análisis simplificado que conduce a resultados muy próximos a los que se obtienen de análisis dinámicos.

En la ref 23 se muestra que una distribución bilineal de la acelera ción a lo alto de la chimenea, cuyas caracteristicas aparecen en la fiğ 4 , 
que al aplicarse estáticamente a la chimenea, permite calcular los momen tos, cortantes y momentos de volteo con suficiente aproximación. Este planteamiento difiere del propuesto en 1 a ref 21 , en donde se establece la necesidad de colocar una concentración en la parte superior de la chimenea.

Cuando las relaciones $i \mathrm{y}$ j se encuentran comprendidas entre los lími tes $0.1<\mathrm{i}<10$ y $1<\mathrm{j}<10$, es necesario efectuar estudios dinámicos que tomen en cuenta la interacción suelo-estructura, a fin de conocer los ele mentos mecánicos a lo largo del fuste y establecer el momento de volteo $\bar{y}$ el cortante en la base. En la ref 24 se muestran las características del análisis y los resultados obtenidos, que indican que el efecto de interac ción puede alterar de manera significativa las características de respues ta de la estructura, dependiendo del tipo de suelo de cimentación y del espectro de diseño seleccionado para analizar a las chimeneas.

Cuando los parámetros i y j son respectivamente inferiores a 0.1 y 10 , es recomendable un cambio en las condiciones de cimentación, ya que los va lores de las constantes de resorte representativas del suelo, no permiten asegurar la estabilidad de la chimenea bajo la acción de la gravedad, por lo que la perturbación inducida en la base por el movimiento sísmico, mos traría la inestabilidad de la estructura, y conduciría a su colapso.

\section{TANQUES ESFERICOS}

Este tipo de estructura, comúnmente empleada para el almacenamiento de líquidos de diversa índole, resulta ser una estructura cuyo comportamiento bajo acción sísmica es poco conocido; por ello, su análisis es poco frecuen te. Al revisar la literatura se encontraron las ref 25 y 26 , que contienen el resultado de análisis, mediciones de campo y mediciones en pruebas de mesa vibradora, que han conducido a un procedimiento de análisis relativa mente simple.

El método \$implificado que permite estimar la respuesta de este tipo de estructuras, considera que el cascarón del tanque y el líquido que contie ne, funcionan como un elemento rígido de masa variable, soportado por una estructura flexible, que juegan un papel importante en el análisis.

E1 método considera al tanque idealizado como un sistema de dos grados de libertad, cuyo comportamiento se asemeja al observado en pruebas de me sa vibradora, $y$ en mediciones de campo, en las cuales los espectros de rés puesta contienen dos frecuencias adicionales a las del espectro del moví miento del terreno.

Uno de los grados de libertad corresponde al movimiento horizontal de la masa del tanque y el líquido que contiene, con una masa equivalente que corresponde al de la pared de acero y a la masa adherida a la pared por el movimiento del tanque. La magnitud de la masa adherida depende la relación existente entre la masa del líquido contenido a la masa total que correspon de al volúmen total de la esfera, def̣inida esa relación mediante el paráme tro $n$. 
Ia constante del resorte correspondiente a esa masa, se obtiene de la estructura de soporte, rígidamente unida a la esfera. Los tirantes y las columnas de la estructura son las que definen a esa constante de resorte.

Cálculos que consideran planteamientos energéticos, o ánalisis matri ciales, que tomen en cuenta los efectos de flexión y fuerza axial, consti tuyen procedimientos cuyos resultados son próximos a los que se obtienen de mediciones experimentales.

E1 segundo grado de libertad representa al movimiento horizontal del agua libre contenida en el depósito (modo de chapoteo), que no se adhiere a la masa de la esfera, la cual provoca oleaje cuya frecuencia se puede calcular a partir de los periodos correspondientes a cada modo:

$$
\begin{aligned}
& T_{1}=2 \pi \sqrt{\left(W_{f}+w_{0}\right) / g k_{f}} \\
& T_{2}=2 \pi \sqrt{w_{s} / g k_{s}}
\end{aligned}
$$

donde

$T_{1}, T_{2}$ los periodos de los modos descritos

$W_{0} \quad$ peso del tanque esférico

$W_{f}$ peso de lamasa adherida del líquido contenido

$W_{S}$ peso del agua libre

$k_{f} \quad$ constante del resorte del primer modo

$k_{5}$ constante del resorte del agua libre

Si se considera que $T_{0}$ es el periodo del tanque sin agua; $B_{f}=\frac{T_{0}}{T_{0}} \quad$ (6.3) $B_{S}=\frac{T_{0}}{T_{2}}(6.4)$ y $M_{f}$ es el factor dinámico de la estructura, definido median te $M_{f}=\frac{1}{1-B_{f}^{2}}+\frac{k_{s} M_{s}}{k_{f}}$

Y $M_{S}=\frac{1}{1-B^{2}}(6.6)$ es el factor dinámico de la masa libre, $y=T_{0} / T_{2}$, se puede establecer que $W_{S}=\left(M_{f}-\frac{1}{1-B_{f}^{2}}\right)\left[\frac{k_{f} T_{2}^{2}}{4 \pi^{2}} g\right]\left(1-B^{2}\right)$

Además, el peso total del líquido contenido, $W_{W^{\prime}}$ debe satisfacerse la condi ción

$$
W_{W}=W_{f}+W_{s} ;\left(\frac{1}{2 \pi}\right)^{2} k_{f} g=W_{f}+W_{0}
$$


De estas expresiones se puede obtener:

$$
\begin{aligned}
& K_{S}=\frac{W_{S}}{g} \frac{4 \pi^{2}}{T_{2}^{2}} \\
& T_{2}=0.134 \sqrt{D} s(n)
\end{aligned}
$$

Siendo

$$
\begin{array}{rl}
D & \text { diámetro del tanque esférico } \\
S_{n} & \doteq 1-\frac{0.313 n}{\sqrt{\frac{W_{f}+W_{0}}{g k_{f}}}}(0.1 \leq n \leq 0.80) \\
T_{1} & =2 \pi \sqrt{W_{f}}=W_{W} f(n) \\
y & f(n) \text { se obtiene de la siguiente tabla }
\end{array}
$$

\begin{tabular}{|c|l|l|l|l|l|l|l|l|l|l|l|}
\hline$n$ & 0 & 0.1 & 0.2 & 0.3 & 0.4 & 0.5 & 0.6 & 0.7 & 0.8 & 0.9 & 1.0 \\
\hline$F(n)$ & 0 & 0.12 & 0.20 & 0.27 & 0.33 & 0.37 & 0.43 & 0.49 & 0.57 & 0.69 & 1.00 \\
\hline
\end{tabular}

Conocidos los periodos naturales de vibración se puede recurrir a es pectros de diseño para definir los desplazamientos y las fuerzas en cada resorte, $y$ as $\not{q}$ obtener los momentos y fuerzas axiales en columnas y la tensión en lo\$ tirantes.

Para estimar la fuerza en el tirante se puede recurrir a la expresión:

$$
F_{T}=\frac{4 L^{2} P}{T_{2}^{2}}-\frac{\Pi^{2} E I}{L^{2}}=\frac{4 L^{2} P}{T_{2}^{2}}-P_{\text {crit }}
$$

donde L la longitud del tirante, $P$ la masa por unidad de longitud del tiran te, I el momento de inercia del tirante, " $F_{T}$ es la fuerza en el tirante.

Esta expresión es de carácter aproximado, para vibración en resonancia en el modo natural $T_{2}$, Se puede aplicar para establecer prediseños, con sidevanco pretensiones en jos tirantes del orden de $\varepsilon=300$ millonésimas en los tirantes, a fin de evitar su trabajo en compresión.

La observación de la vibraoión de estructuras de tanques esféricos ha mostrado que se trata de estructuros cuya estabilidad depende principalmen 
te de las conexiones entre columnas y tirantes ya que las mediciones de deformaciones unitarias han mostrado concentraciones importantes de ellas en las uniones.

Las pruebas en mesa vibradora de modelos a escala indican que la placa de la esfera no experimenta deformaciones unitarias importantes.

\section{COMENTARIOS FINALES}

La revisión de los daños en estructuras industriales, inducidos por sismos, y de los criterios empleados para su análisis sísmico, muestra que las estructuras de tanques, torres y altos hornos son estructuras rígi das de baja ductilidad, cuyo comportamiento bajo la acción de sismos inten sos depende de las características de la cimentación y de las anclas de unión de la estructura a sus bases. A fin de evitar su volteo se debe poner especial cuidado en la estimación del cortante sísmico, así como en los mo mentos de volteo, en la base.

Las chimeneas son estructuras flexibles, que requieren de cuidados es peciales en su diseño, dada la alta incidencia de daños en ellas durante sismos intensos; por ello, se debe prestar especial atención a las condi ciones de la cimentación, su rigidez y la relación de rigideces entre los fustes y el suelo, a fin de considerar correctamente la acción sísmica.

Se puede considerar que en el diseño de este tipo de estructuras bajo la acción sísmica, es necesario visualizar a la estructura, su cimentación y el suelo, como una sola parte estructural y garantizar las condiciones de unión y de resistencia que permitan establecer la estabilidad de estas estructuras, bajo la acción de sismos intensos.

Los criterios de diseño revisados permiten estimar las principales acciones que se producen durante sismos, así como estimar las acciones di námicas que definen a los elementos mecánicos de diseño de la estructura y su anclaje.

En aquellas estructuras que contienen líquidos, el uso de métodos de elemento finito permite estimar la acción de la interacción entre el reci piente y el líquido, tanto para la revisión de las paredes y sus conexiones, como para estimar correctamente el incremento de momento de volteo sobre la cimentación, resultante de los efectos dinámicos generados por el movimien to del fluído en el interior de los recipientes.

Para tanques esféricos, esta acción resulta importante para el diseño de la estructura y tirantes de soporte, ya que el cascarón esférico se com porta como un elemento rígido, si satisface los criterios comunes de dise ño empleados para soportar su peso y las presiones que provoca el líquido sobre las paredes, $y$ se refuerza adecuadamente su conexión con la estructú - ra de soporte. 


\section{REFERENCIAS}

1. Zhong-quan, X. "Aseismic Design of Towers, Tanks and Furnaces", Publicación 80-c2/PVP-87. American Society of Mechanical Engineers. Presen tado en una conferencia en San Francisco, Cal. Agosto 1980

2. Butler, R. Stewart G. y Kanamori, H., "The July 27, 1976 Tangshan, China Earthquake A Complex Sequence of Intraplate Events". Bull. Seis. Soc. of America, Vol 69, No 1, pp 207-220, Febrero 1979

3. Housner, G W, "Dynamic Pressures on Accelerated Fluid Containers". Bulletin of the Seis. Soc. of America, 47, 1957

4. Zhong-quan, $\mathrm{X} y$ coautores. "Dynamic pressure Analysis of Offshore Platforms During Earthquake". Institute of Construction Technology, Minis try of Petroleoum Industry, China, Mayo 1978.

5. Veletsos, A. S. "Seismic Effects in Flexible Storage Tanks". Proc V Conferencia Mundial de Ingeniería Sísmica, 1972

6. Flores, R. "Design Principles of Earthquake Resistant Blast Furnaces". Proc. III Conferencia Mundial de Ingeniería Sísmica, 1965

7. Anónimol "Japanese Furnace is Earthquake Proof". Steel. Vol 156, No 4,1965

8. Miyo Shima Chino. "Aseismic Design of Blast Furnace". $\mathrm{N}^{\circ} 59,1976$, pp 1

9. Scholl, R. "Statistical Anaysis of Low-rise Building Damage Caused by the San Fernando Earthquake". Bull. Seis. Soc of America. Vol 64, 1974, pp 1-23

10. Comité Estatal de Construcción del Consejo de Ministros de la URSS. "Standard Regulations for Building in Seismic Regions. (SN-8-57)". Agosto 9 1957

11. Structural Engineers Association of California. "Recommended Lateral Force Requirements and Commentary". San Francisco, 1967

12. Rinne, J E. "Design of Earthquake-Resistant Structures: Towers and Chimneys" en "Earthquake Engineering" por R. Wiegel; Capítulo 20, pp 495-505

13. Applied Technology Council. "Tentative Provisions for the Develop ment of Seismic Regulations for Buildings". NBS Special Publication 510, Junio 1978, Capítulo 9, pp 77-84 
14. Balendra, Ty Nash, W A. "Seismic Analysis of a Cylindrical Liquid Storage Tank with a Dome, by the Finite Element Method". Publicación ASME 80-C2/PUP-74. San Francisco Cal, Agosto 1980

15. Gould, P L y Seng-Lip L. "Hyperbolic Cooling Towers under Seismic Design Load". Artículo 5268. Journal of the Structural Division. ASCE, ST3, Junio 1967, pp 87-109

16. Gould, P L. "Seismic Design of Hyperbolic Cooling Towers", en "Structural Design of Nuclear Plant Facilities". ASCE. Nueva York, 1975

17. Gould, P L y Abu-Sitta, SH. "Dynamic Response of Structural to Wind and Earthquake Loading". Halsted Press Book. Nueva York, 1980

18. Abel, J F, Cole, P B y Billington, D B. "Maximum Seismic Response of Cooling Towers". Informe de Investigación 73-SM-1. Princeton University, 1973

19. Gould, P I. Sen, SK y Suryoutomo, H. "Dynamic Analysis of Columnsupported Hyperboloidal Shells". Earth Eng. and Struct. Dynamics. Vol 2. 1974, pp 269-279

20. Ciesielki, R y coautores. "Behälter, Bunker, Silos, Shornsteine, Fernsehturme und Freileitungsmaste". W. Ernst \& Sons. 1970

21. ACI Standard 307. "Specifications for the Design and Construction of Reinforced Concrete Chimneys".

22. British Standards Institution: "Specification for Steel Chimneys" BS 4076: 1978 Londres, 1978

23. Rodríguez Cuevas, N. "Torres y chimeneas". VII Curso Internacional de Ingeniería Sísmica. UNAM. Agosto 1981

24. Aranda, G R. "Soil Effects on Seismic Response of Chimneys". Publi cación E-45. Instituto de Ingeniería, UNAM. Enero de 1981

25. Ando, $\mathrm{K}$ y Shimosaka, S. "Measurements and response Analysis of the Seismic Behavior of a Spherical Tank". Engineering Review. Vol 13, No 1. Enero 1980, pp 1-6

26. Nakano, $\mathrm{K}$ y Watanabe, M. "Experimental Research on the Aseismic Characteristics of Spherical steel Tank for Liquid Petroleum Gas". Proc. VI Joint UJNR Panel Conference. NBS Special Publication 444. Abril 1976, pp V50-V-62. 


$$
V=Z K C W
$$

$\checkmark$ cortante en la base

$Z$ coeficiente de sismicidad

$K$ coeficiente lde estructura $=2.0$ para torres

C coeficiente de flexibilidad $=0.05(T)^{-1 / 3}$

$W$ peso de la estructura en operación

$F_{t}=0.004 V\left(h_{n} / D\right)^{2}$

$F_{t}=0$ cuando $\left(h_{n} / D\right)<3$

$F_{t}=0.15 \mathrm{~V}$, cuanido $\left(h_{n} / D\right)>6.12$

$F_{x}=\left(V-F_{t}\right) \frac{w_{x} h_{x}}{\sum \sum_{i}^{n} w_{i}}$

$v=F_{t}+\sum_{i=1}^{n} F_{i}^{i-1}$

$v_{x}=F_{t}+\sum_{i=1}^{n} F_{i}$

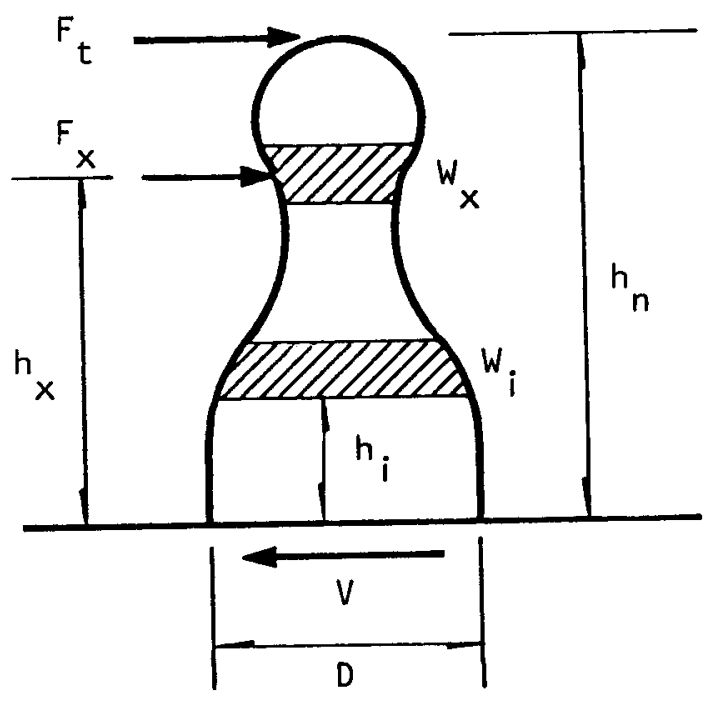

a) Distribución de cortantes

$M=J\left(F_{t} h_{n}+\sum_{i=1}^{n} F_{i} h_{i}\right)$

$J=0.6 \mathrm{~T}^{-1 / 3}$

$T=$ periodo fundamental, en seg

$M_{x}=J_{x}\left[F_{t}\left(h_{n}-h_{x}\right)+\sum_{i=1}^{n} F_{i}\left(h_{i}-h_{x}\right)\right]$

$J_{x}=J+(1-J)\left(h_{x} / h_{n}\right)^{3}$

$J_{x}=J=1.0$ cuando $T \overline{<} 0.216 \mathrm{seg}$

$J_{\min }=0.45$ para estructuras industriales

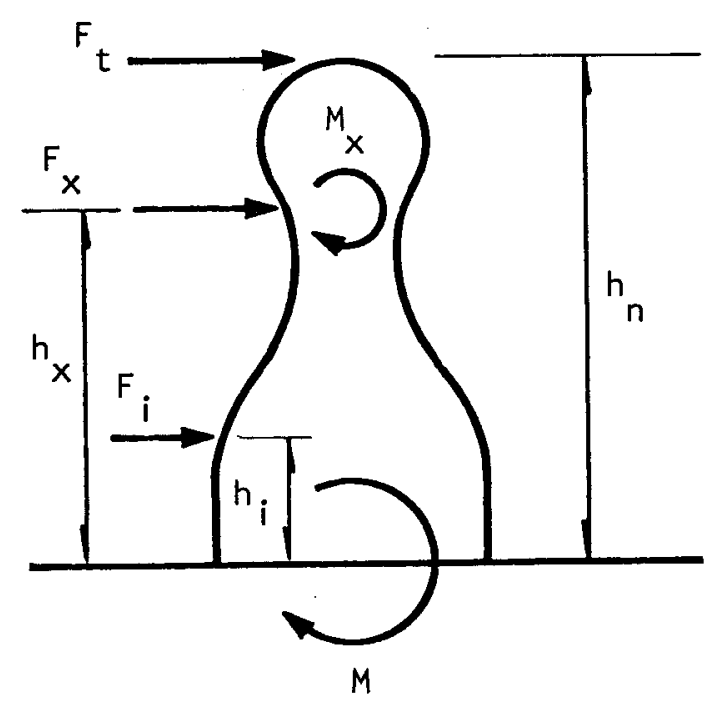

b) Distribución de momentos

Fig 1. Recomendaciones SEAOC para la determinacion de acciones sísmicas en torres $y$ chimeneas 


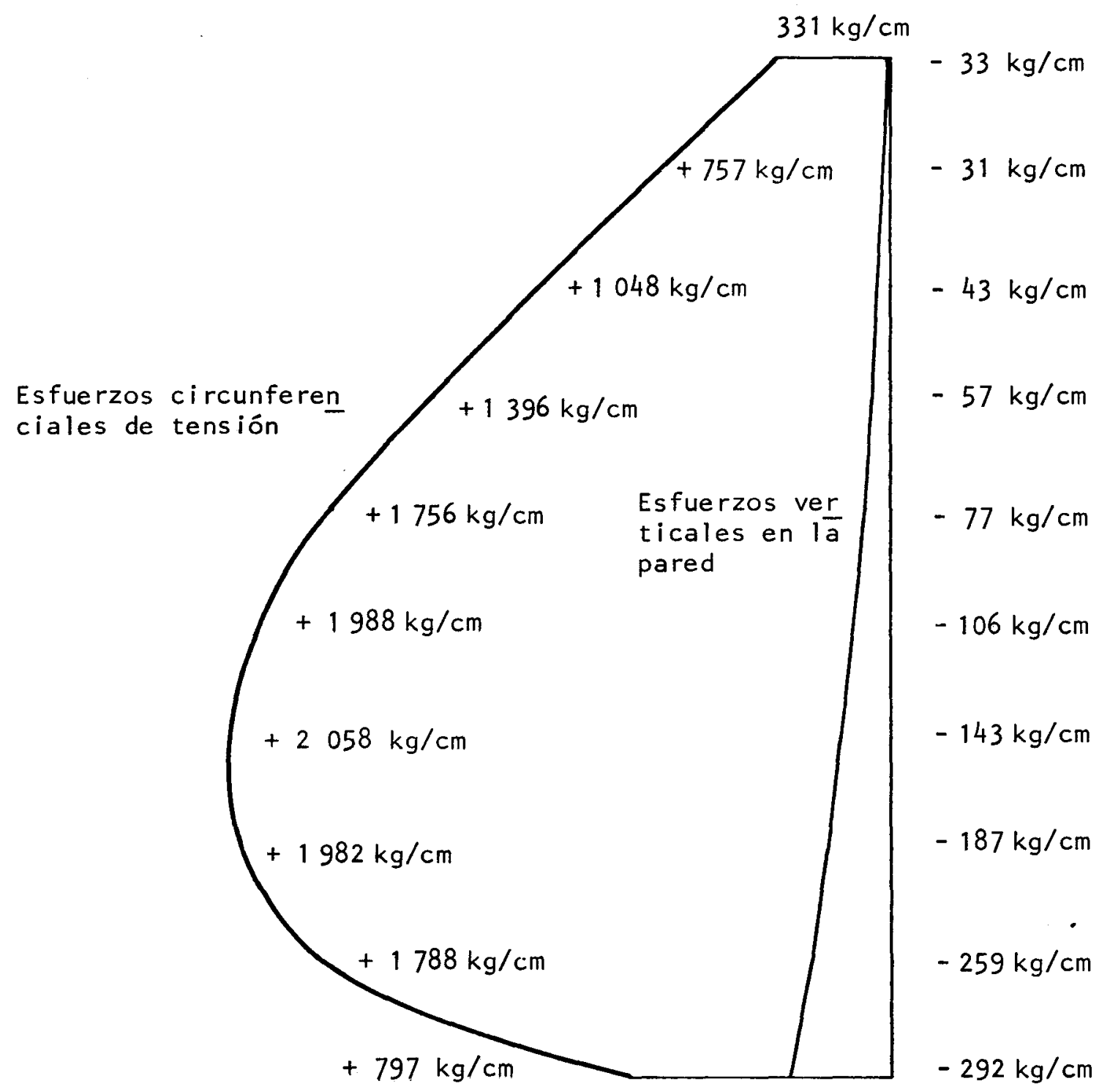

Fig 2. Distribución de fuerzas de membrana en la pared de un tanque cubierto de $36.58 \mathrm{~m}$ de diametro, bajo un sismo registrado, con aceleración máxima igual a $0.5 \mathrm{~g}$ 


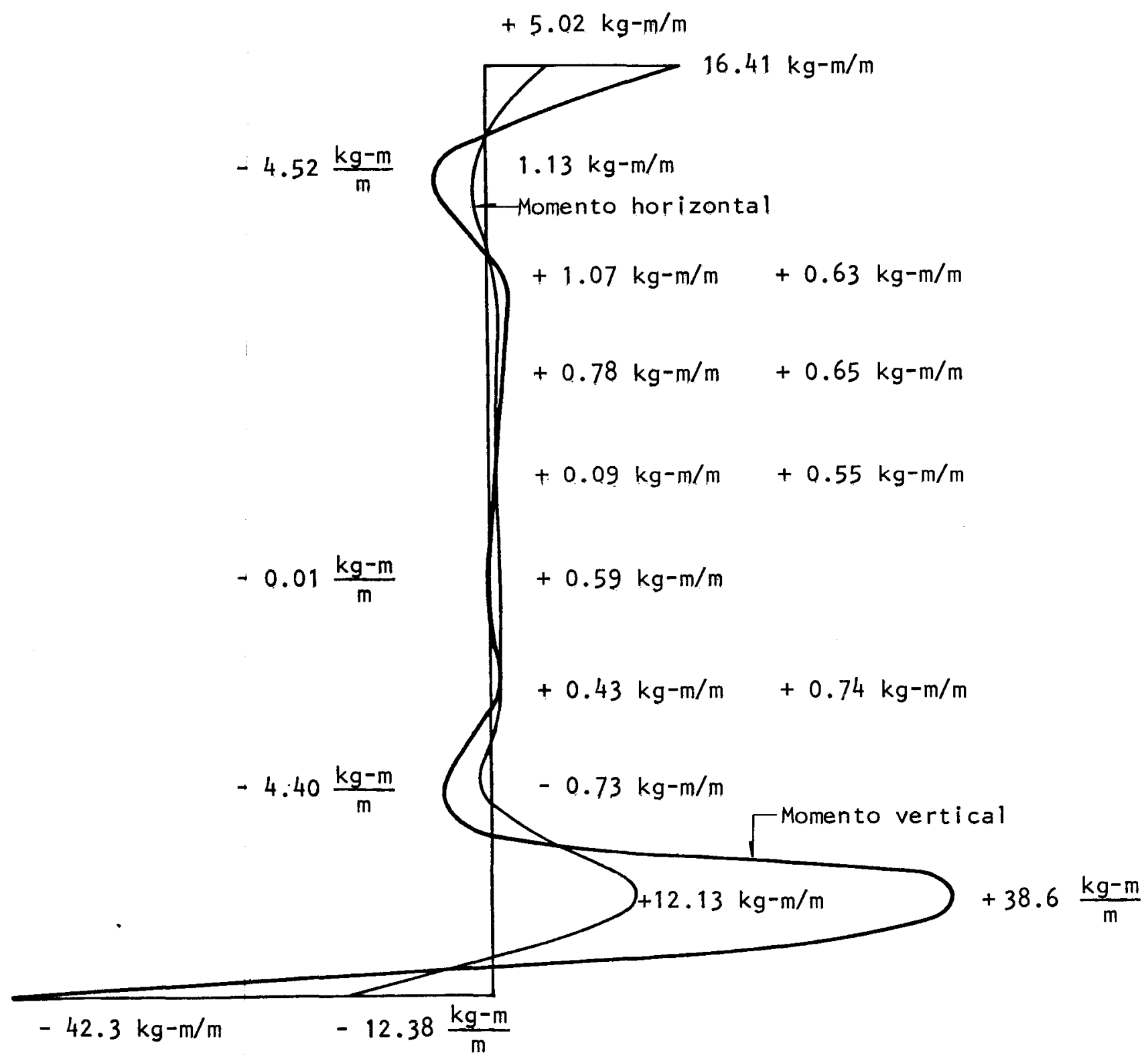

Fig 3. Distribución de momentos flexionantes unitarios en la pared vertical de un tanque de $36.58 \mathrm{~m}$, bajo un sismo registrado, con aceleración maxima igual a $0.5 \mathrm{~g}$ 


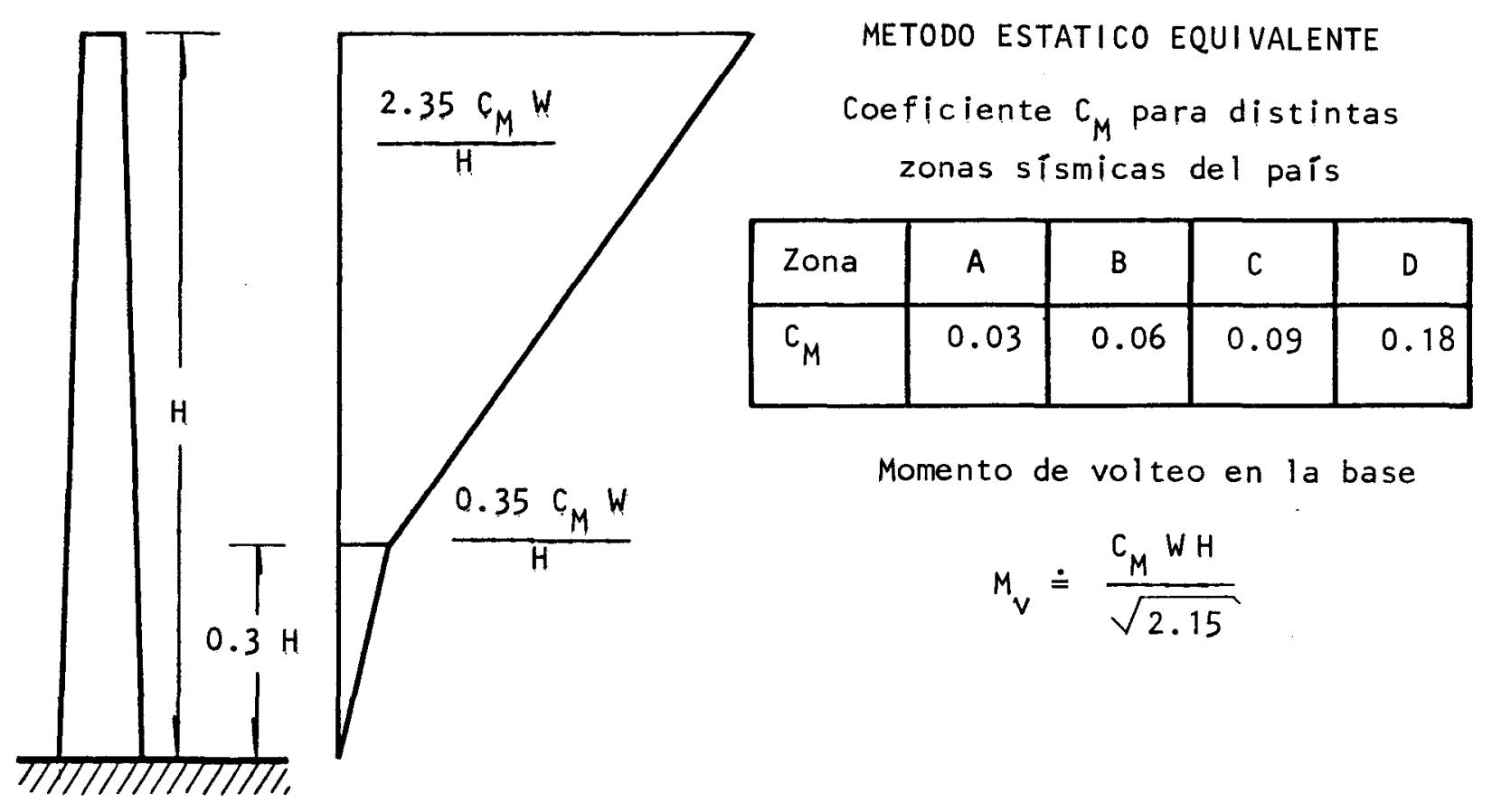

Resultados obtenidos de aplicar el método a una chimenea de $80 \mathrm{~m}$ en zona $D$

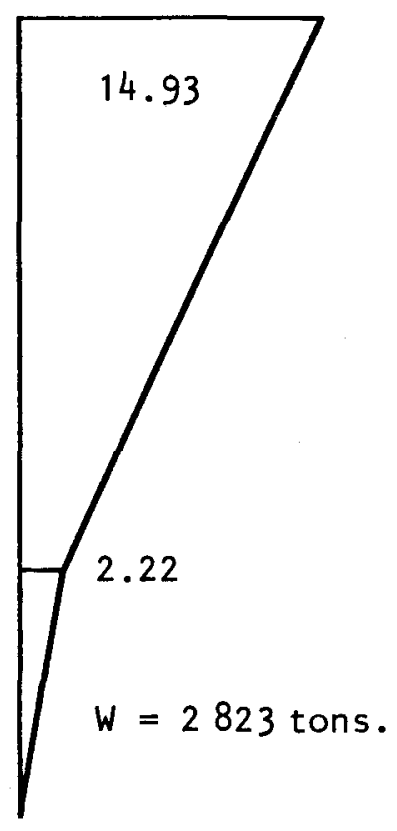

Carga lateral equivalente $($ ton $/ \mathrm{m})$

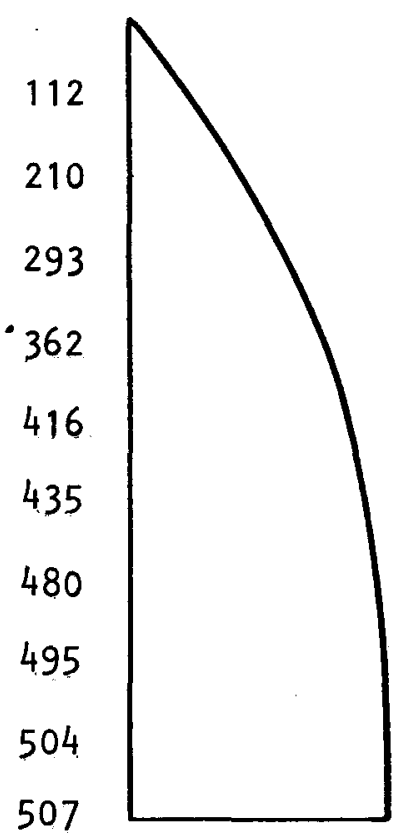

Fuerza cortante (ton)

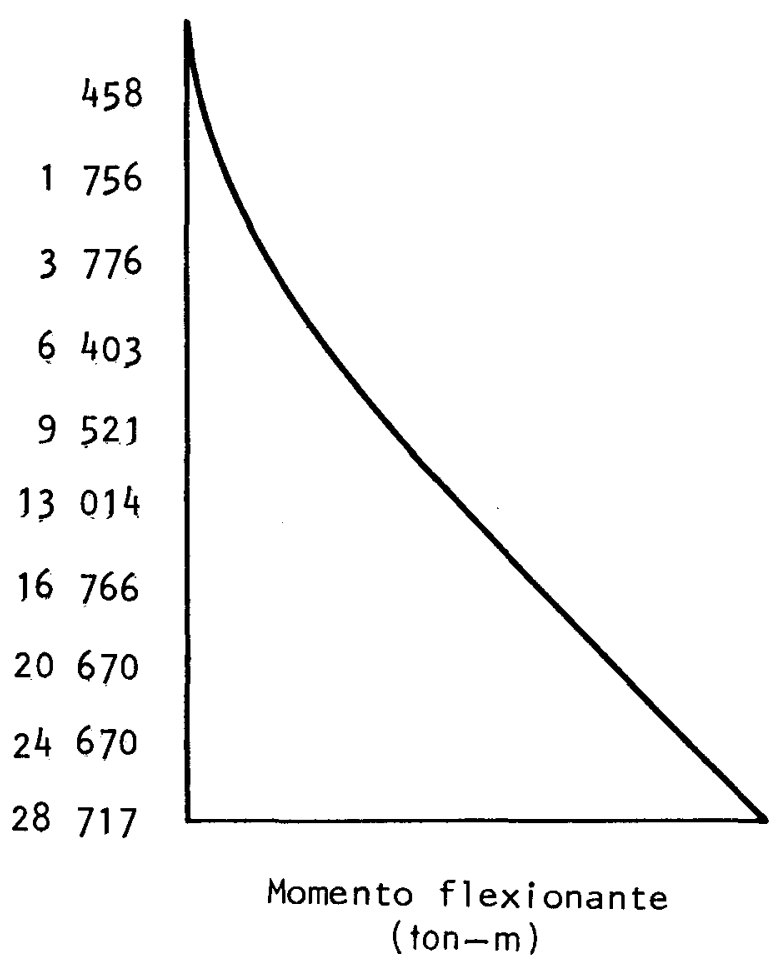

$($ ton $-\mathrm{m})$

Fig.4. Metodo estatico equivalente para analisis sismico de chimeneas, en una cimentación en la que los parámetros $i$ y son superiores a 10 